archives-ouvertes

\title{
Heat and mass transfer in a rarefied gas confined between its two parallel condensed phases
}

\author{
Alexey Ph Polikarpov, Irina Graur
}

\section{To cite this version:}

Alexey Ph Polikarpov, Irina Graur. Heat and mass transfer in a rarefied gas confined between its two parallel condensed phases. International Journal of Heat and Mass Transfer, Elsevier, 2018, 124, pp.967-979. 10.1016/j.ijheatmasstransfer.2018.04.006 . hal-01782351

\section{HAL Id: hal-01782351 \\ https://hal.archives-ouvertes.fr/hal-01782351}

Submitted on 22 Jul 2020

HAL is a multi-disciplinary open access archive for the deposit and dissemination of scientific research documents, whether they are published or not. The documents may come from teaching and research institutions in France or abroad, or from public or private research centers.
L'archive ouverte pluridisciplinaire HAL, est destinée au dépôt et à la diffusion de documents scientifiques de niveau recherche, publiés ou non, émanant des établissements d'enseignement et de recherche français ou étrangers, des laboratoires publics ou privés. 


\title{
Heat and mass transfer in a rarefied gas confined between its two parallel condensed phases
}

\author{
Alexey Ph Polikarpov ${ }^{1}$, Irina A. Graur ${ }^{2}$ \\ ${ }^{1}$ Ural Federal University, 51 str. Lenina, 620000 Ekaterinbourg, Russia \\ ${ }^{2}$ Aix-Marseille Université, CNRS, IUSTI UMR 7343, 13013 Marseille, France
}

\begin{abstract}
The heat and mass transfer in a rarefied gas between its two parallel condensed phases is considered on the basis of linearized and non-linear S-model kinetic equations. The profiles of the macroscopic parameters in the gap between gas-liquid interfaces are obtained for several Knudsen numbers and for the cases of complete and non-complete evaporation and condensation. The linearized Navier-Stokes equations and energy equation, subjected to the temperature and pressure jump boundary conditions, are solved analytically and the expressions for the macroscopic parameters are obtained. The comparison of three approaches allowed us to establish the limit of the application of the linearized approaches in term of the saturation temperature ratio between the condensed phases.
\end{abstract}

\section{Introduction}

Evaporation and condensation phenomena are interesting not only from the fundamental point of view, but they are also largely present in various industrial applications. During the evaporation and condensation the liquid-vapor interface is in non-equilibrium state and its detailed modeling requires the use of the molecular based approaches, like the kinetic theory of gases [1] or the molecular dynamics [2]. The adequate description of the evaporation-condensation was in focus of interest from a long time [3], [4], [5], [6], [7], [8], [1]. However, the complete understanding of these phenomena is just far to be completely achieved. In addition, the implementation of the kinetic models or the molecular dynamics based approaches is not so easy for the practical problems. This is why it is also interesting to use the continuum approaches, like the classical Navier-Stokes equations, subjected with the temperature and pressure jumps boundary conditions [9], [10] to simulate the evaporation-condensation problems. Recently the alternative macroscopic approach based on the regularized 13 moments equations [11] was also proposed and applied for the simulation of the evaporation and condensation phenomena. 
The main objective of this study is the development of the numerical approach to simulate the behavior of the vapor phase between two parallel liquidvapor interfaces. Contrarily to the authors of Ref. [8], where the BGK equation is implemented, the linearized and non-linear S-model [12] kinetic equations are solved numerically to simulate the evaporation-condensation phenomena appearing between these two plane condensed phases. The main advantage of the S-model is its capacity to provide the correct Prandtl number, equals to $2 / 3$, for the monatomic gases, which can be important, when the problems involving the vapor flows and the heat flux are considered. In addition, the linearized Navier-Stokes equations and energy equation subjected to the temperature and pressure jumps boundary conditions [9], [10] are also solved analytically and the simple relations are obtained for the profiles of the macroscopic param-

eters of vapor in the gap between two condensed phases. The influence of evaporation-condensation coefficient on the macroscopic parameters is also analyzed. Comparing the results obtained by three approaches we establish the limit of the applicability of the linearized kinetic equation as well as of the linearized Navier-Stokes equations for the simulation of the evaporation and condensation phenomena.

\section{Problem statement}

We consider two parallel plane condensed phases at rest, maintained at temperature $T_{1}$ and $T_{2}$, on the bottom $\left(y^{\prime}=0\right)$ and top $\left(y^{\prime}=H\right)$ interfaces, respectively, $y^{\prime}$ is the coordinate normal to both condensed phases. Let $p_{1}$ and $p_{2}$ be the saturation gas pressures at temperatures $T_{1}$ and $T_{2},\left(T_{1}>T_{2}\right)$, respectively. We investigate here the behavior of the monatomic gas motion caused by evaporation and condensation on the condensed phases, first on the basis of the kinetic theory.

If we consider that the ratios $p_{1} / p_{2}$ and $T_{1} / T_{2}$ are independent, then the evaporation-condensation problem is completely characterized by three parameters: $p_{1} / p_{2}$ and $T_{1} / T_{2}$ ratios and by the rarefaction parameter $\delta$. However, in practice, the saturation pressure and temperature are related by the ClausiusClapeyron equation [13] and therefore cannot take the arbitrary values. In this case the evaporation-condensation problem depends on two parameters only: the slop of $p^{\prime}\left(T^{\prime}\right)$ function and the rarefaction parameter $\delta$. In the following we consider both cases: first the situation when two parameters are independent, and then, the cases of Argon and several other monatomic gases, where the saturation pressure and temperature are related through the Clausius-Clapeyron equation.

The distance $H$ between two interfaces is taken as the characteristic dimension of the problem, so the rarefaction parameter $\delta$ is defined as following:

$$
\delta=\frac{H}{\ell}
$$




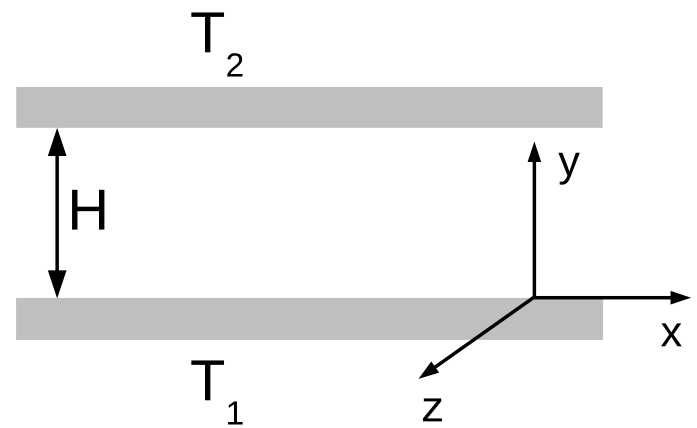

Figure 1: Sketch of 1D geometry

where $\ell$ is the equivalent mean free path:

$$
\ell=\frac{\mu_{2} v_{2}}{p_{2}},
$$

which is defined using the reference parameters with subscript 2, corresponding to the upper plate. The choice of the parameters related to the upper plate as the reference parameters was done without lost of generality. In Eq. (2) $\mu_{2}=\mu\left(T_{2}\right)$ is the dynamic viscosity of the vapor phase

$$
\mu\left(T^{\prime}\right)=\mu_{2} \sqrt{\frac{T^{\prime}}{T_{2}}},
$$

$v_{2}$ is the most probable molecular speed,

$$
v\left(T^{\prime}\right)=\sqrt{2 \mathcal{R} T^{\prime}},
$$

calculated at the temperature $T_{2}: v_{2}=v\left(T_{2}\right), \mathcal{R}=k_{\mathrm{B}} / m$ is the specific gas constant, $k_{\mathrm{B}}$ is the Boltzmann constant, $m$ is the molecular mass.

\section{Modeling based on the kinetic equation}

To model the evaporation and condensation phenomena between two parallel condensed phases maintained at different temperatures first the S-model kinetic equation [12] is used. The evaporation-condensation problem between two parallel condensed phases is considered here as steady state and one dimensional in physical space, so the S-model kinetic equation is written as

$$
v_{y} \frac{\partial f}{\partial y^{\prime}}=v\left(f^{S}-f\right),
$$

where $f\left(y^{\prime}, \mathbf{v}\right)$ is the one particle velocity distribution function, $\mathbf{v}=\left(\mathbf{v}_{\mathbf{x}}, \mathbf{v}_{\mathbf{y}}, \mathbf{v}_{\mathbf{z}}\right)$ is the molecular velocity vector, $v$ is the collision frequency, $v=p^{\prime} / \mu$. In the 
frame of S-model the equilibrium distribution function $f^{S}$ in Eq. (5) is defined as following

$$
f^{S}\left(y^{\prime}, \mathbf{v}\right)=f^{M}\left[1+\frac{2 m \mathbf{V} \mathbf{q}^{\prime}}{15 n^{\prime}\left(y^{\prime}\right)\left(k_{\mathrm{B}} T^{\prime}\left(y^{\prime}\right)\right)^{2}}\left(\frac{m \mathbf{V}^{2}}{2 k_{\mathrm{B}} T^{\prime}\left(y^{\prime}\right)}-\frac{5}{2}\right)\right],
$$

here $T^{\prime}\left(y^{\prime}\right)$ is a gas temperature, $n^{\prime}\left(y^{\prime}\right)$ is a gas number density, $\mathbf{u}^{\prime}=\left(0, u^{\prime}, 0\right)$ is a bulk velocity vector, $\mathbf{V}=\mathbf{v}-\mathbf{u}^{\prime}$ is the peculiar velocity vector, $\mathbf{q}^{\prime}=\left(0, q^{\prime}, 0\right)$ is a heat flux vector, $f^{M}$ is the Maxwellian distribution function [14]. The macroscopic parameters are defined as follows:

$$
\begin{aligned}
& n^{\prime}\left(y^{\prime}\right)=\int f\left(y^{\prime}, \mathbf{v}\right) \mathrm{d} \mathbf{v}, \quad u^{\prime}\left(y^{\prime}\right)=\frac{1}{n} \int f\left(y^{\prime}, \mathbf{v}\right) v_{y} \mathrm{~d} \mathbf{v}, \\
& T^{\prime}\left(y^{\prime}\right)=\frac{m}{3 k_{\mathrm{B}} n^{\prime}} \int f\left(y^{\prime}, \mathbf{v}\right) V^{2} \mathrm{~d} \mathbf{v}, \quad q^{\prime}\left(y^{\prime}\right)=\frac{m}{2} \int f\left(y^{\prime}, \mathbf{v}\right) V^{2}\left(v_{y}-u_{y}^{\prime}\right) \mathrm{d} \mathbf{v}(8)
\end{aligned}
$$

By integrating Eq. (5) multiplied by $\left(1, v_{y}, v_{i}^{2}\right)$ we obtain the integrals expressing the number of molecules, $y$ component of momentum and energy transported in $y$ direction per a unit area of a plan, parallel to the condensed phases. The evaporation flow rate, expressed in the number of molecules per time and per surface unit, $J_{n}^{\prime}$, and the evaporation mass flow rate, expressed in kilogram per time and surface units, $J_{\rho}^{\prime}$, are defined as followings:

$$
J_{n}^{\prime}=\int v_{y} f\left(y^{\prime}, \mathbf{v}\right) \mathrm{d} \mathbf{v}, \quad J_{\rho}^{\prime}=m \int v_{y} f\left(y^{\prime}, \mathbf{v}\right) \mathrm{d} \mathbf{v} .
$$

The second definition of the evaporation mass flow rate is usually provided from the experiments. The energy flux is defined as

$$
J_{E}^{\prime}=\int v_{y} v^{2} f\left(y^{\prime}, \mathbf{v}\right) \mathrm{d} \mathbf{v} .
$$

The constancy of the integrals $J_{n}^{\prime}$ and $J_{E}^{\prime}$ will be further used to estimate the accuracy of the numerical calculations and for the comparison with the results of Ref. [8].

\subsection{Linearized S-model equation}

To linearize S-model kinetic equation (5) we assume that deviations between the temperatures of two condensed surfaces and the corresponding saturation pressures are small:

$$
X_{P}=\frac{p_{1}-p_{2}}{p_{2}} \ll 1, \quad X_{T}=\frac{T_{1}-T_{2}}{T_{2}} \ll 1 .
$$

In previous expressions $X_{P}$ and $X_{T}$ can be related to the thermodynamic forces [15]. As it was mentioned in Section 2, for a given gas the pressure and temperature differences are coupled by the relation

$$
p_{1}-p_{2}=\beta\left(T_{1}-T_{2}\right),
$$


where $\beta$ is a positive constant corresponding to the slop of the Clausius-Clapeyron curve at $T_{2}$, so $X_{P}$ and $X_{T}$ are not independent quantities. However here we consider two forces separately, to see clearly the impact of each force to the evaporation-condensation process.

For further derivation we introduce the following dimensionless quantities:

$$
y=\frac{y^{\prime}}{H}, \quad \mathbf{c}=\frac{\mathbf{v}}{v_{2}}, \quad \mathbf{u}=\frac{\mathbf{u}^{\prime}}{v_{2}}, \quad n=\frac{n^{\prime}}{n_{2}}, \quad T=\frac{T^{\prime}}{T_{2}}, \quad p=\frac{p^{\prime}}{p_{2}}, \quad \mathbf{q}=\frac{\mathbf{q}^{\prime}}{p_{2} v_{2}} .
$$

When $X_{P}$ and $X_{T}$ are small enough compared to 1 we can linearize the distribution function as following:

$$
f=f_{0}^{M}\left(1+h_{P} X_{P}+h_{T} X_{T}\right),
$$

where $h_{P}$ and $h_{T}$ are the perturbation functions, related to the pressure and temperature difference, respectively, $f_{2}^{M}$ is the absolute Maxwellian distribution function with the reference number density, $n_{2}$ and temperature, $T_{2}$ :

$$
f_{2}^{M}=n_{2}\left(\frac{m}{2 \pi k_{\mathrm{B}} T_{2}}\right)^{3 / 2} \exp \left(-c^{2}\right)
$$

For the real gases, for any value of $\beta$ we can obtain the solution as a superposition of two functions as

$$
h=\left(\beta h_{P}+h_{T}\right) \Delta T,
$$

where $\Delta T=T_{1}-T_{2}$. However, as it was mentioned above, we consider here $X_{P}$ and $X_{T}$ as independent quantities.

The S-model kinetic equation (5) in its linearized form can be separated into two equations with reference to the thermodynamic forces $X_{P}$ and $X_{T}$ :

$$
c_{y} \frac{\partial h_{i}}{\partial y}=\delta\left(\nu_{i}+2 c_{y} u_{i}+\left(c^{2}-\frac{5}{2}\right)\left(\tau_{i}+\frac{4}{15} q_{i} c_{y}\right)-h_{i}\right), \quad i=P, T .
$$

The macroscopic parameters in Eq. (17) can be calculated through the following relations:

$$
\begin{array}{ll}
n=1+\nu_{P} X_{P}+\nu_{T} X_{T}, & u=u_{P} X_{P}+u_{T} X_{T}, \\
T=1+\tau_{P} X_{P}+\tau_{T} X_{T}, & q=q_{P} X_{P}+q_{T} X_{T} .
\end{array}
$$

The dimensionless moments of the distribution function are given as:

$$
\begin{aligned}
\nu_{i} & =\frac{1}{\pi^{3 / 2}} \int \exp \left(-c^{2}\right) h_{i} \mathrm{~d} \mathbf{c}, \\
u_{i} & =\frac{1}{\pi^{3 / 2}} \int \exp \left(-c^{2}\right) h_{i} c_{y} \mathrm{~d} \mathbf{c}, \\
\tau_{i} & =\frac{1}{\pi^{3 / 2}} \int \exp \left(-c^{2}\right) h_{i}\left(\frac{2}{3} c^{2}-1\right) \mathrm{d} \mathbf{c}, \\
q_{i} & =\frac{1}{\pi^{3 / 2}} \int \exp \left(-c^{2}\right) h_{i} c_{y}\left(c^{2}-\frac{5}{2}\right) \mathrm{d} \mathbf{c}, \quad i=P, T .
\end{aligned}
$$


The distribution function of the molecules outgoing from the condensed phases is supposed to be decomposed into two parts [8]: $\sigma_{e}$ part of molecules evaporates from the surface with saturation parameters while another part, $\left(1-\sigma_{c}\right)$, reflects diffusively from the surface. We assume here that the evaporation $\sigma_{e}$ and the condensation $\sigma_{c}$ coefficients are equal each to other $\sigma=\sigma_{e}=\sigma_{c}$.

The linearized form of evaporation-condensation boundary conditions reads:

for the bottom surface, $y=0$ :

$$
\begin{gathered}
h_{P}=\sigma+(1-\sigma) \nu_{P_{w}}, \quad h_{T}=\sigma\left(c^{2}-\frac{5}{2}\right)+(1-\sigma)\left[\left(c^{2}-\frac{3}{2}\right)+\nu_{T_{w}}\right], \\
\nu_{P_{w}}=-\frac{2}{\pi} \int_{c_{y}<0} h_{P} c_{y} \mathrm{~d} \mathbf{c}, \quad \nu_{T_{w}}=-\frac{1}{2}-\frac{2}{\pi} \int_{c_{y}<0} h_{T} c_{y} \mathrm{~d} \mathbf{c}
\end{gathered}
$$

and upper surface, $y=1$ :

$$
\begin{gathered}
h_{P}=(1-\sigma) \nu_{P_{w}}, \quad h_{T}=(1-\sigma) \nu_{T_{w}}, \\
\nu_{P_{w}}=\frac{2}{\pi} \int_{c_{y}>0} h_{P} c_{y} \mathrm{~d} \mathbf{c}, \quad \nu_{T_{w}}=\frac{2}{\pi} \int_{c_{y}>0} h_{T} c_{y} \mathrm{~d} \mathbf{c} .
\end{gathered}
$$

To reduce the number of variables in a velocity space we use the standard procedure [16]: we multiplied first Eqs. (17) successively by 1 and $\left(c_{z}^{2}-\frac{1}{2}\right)$, and then we integrated them over $c_{z}$ velocity. Doing so we obtain the system of two equations for reduced distribution functions:

$$
\begin{gathered}
c_{y} \frac{\partial \phi_{i}}{\partial y}=\delta\left(\nu_{i}+2 c_{y} u_{i}+\left(c_{x}^{2}+c_{y}^{2}-1\right) \tau_{i}+\frac{4}{15} q_{i} c_{y}\left(c_{x}^{2}+c_{y}^{2}-2\right)-h_{i}\right), \\
c_{y} \frac{\partial \psi_{i}}{\partial y}=\delta\left(\frac{1}{2} \tau_{i}+\frac{2}{15} q_{i} c_{y}\right), \quad i=T, P
\end{gathered}
$$

where these reduced distribution functions read:

$$
\begin{aligned}
\phi_{i} & =\frac{1}{\sqrt{\pi}} \int_{-\infty}^{\infty} \exp \left(-c_{z}^{2}\right) h_{i} \mathrm{~d} c_{z}, \\
\psi_{i} & =\frac{1}{\sqrt{\pi}} \int_{-\infty}^{\infty} \exp \left(-c_{z}^{2}\right)\left(c_{z}^{2}-\frac{1}{2}\right) h_{i} \mathrm{~d} c_{z}, \quad i=T, P .
\end{aligned}
$$

The system of equations (28), (29) with the boundary conditions, Eqs. (24) (27), is solved by the Discrete Velocity Method (DVM) [17], see Section 4.

\subsection{Thermodynamic analysis}

The author of Refs. [18], [15] have obtained the Onsager-Casimir reciprocity relation in general form for rarefied gas flows. It was shown [15] that for the case 
of evaporation-condensation of a gas between two parallel condensed phases the "fluxes" corresponding to the driving "forces" are defined as follows:

$$
J_{P}^{\prime}=n_{2} u^{\prime}, \quad J_{T}^{\prime}=\frac{1}{k_{\mathrm{B}} T_{2}} q^{\prime},
$$

where $u_{y}^{\prime}$ and $q_{y}^{\prime}$ do not depend on $y^{\prime}$. The thermodynamic fluxes are related to the thermodynamic forces in the matrix form:

$$
\left[\begin{array}{c}
J_{P}^{\prime} \\
J_{T}^{\prime}
\end{array}\right]=\left[\begin{array}{ll}
\Lambda_{P P} & \Lambda_{P T} \\
\Lambda_{T P} & \Lambda_{T T}
\end{array}\right] \times\left[\begin{array}{c}
X_{P} \\
X_{T}
\end{array}\right] .
$$

In this case, the Onsager-Casimir relation, $\Lambda_{P T}=\Lambda_{T P}$, yields the coupling between the mass flux caused by temperature drop and the thermal flux caused by the pressure drop [15].

\subsection{Non-linear S-model equation}

To establish the limit of applicability of linearized S-model kinetic equation to model two condensed surfaces problem we solve numerically the non-linear S-model kinetic equation. By using Eqs. (13) the dimensionless form of Eq. (5) reads:

$$
c_{y} \frac{\partial f}{\partial y}=\delta n \sqrt{T}\left(f^{S}-f\right) .
$$

As for the linearized case, see Section 3.1, we assume that only one part $\sigma$ of the incident molecules evaporates immediately and $(1-\sigma)$ part reflects from the surface diffusively. Therefore the dimensionless boundary conditions for the distribution function of the reflected molecules at the liquid-gas interfaces can be written as

$$
\begin{aligned}
& y=0 \quad c_{y}>0, \\
& f=\left(\sigma n_{s_{1}}+(1-\sigma) n_{r_{1}}\right) f_{1}^{M}, \quad f_{1}^{M}=\frac{1}{\pi^{3 / 2}} \frac{p_{1}}{p_{2}}\left(\frac{T_{2}}{T_{1}}\right)^{5 / 2} \exp \left(-\frac{T_{2}}{T_{1}} c^{2}\right) \\
& y=1 \quad c_{y}<0, \\
& f=\left(\sigma n_{s_{2}}+(1-\sigma) n_{r_{2}}\right) f_{2}^{M}, \quad f_{2}^{M}=\frac{1}{\pi^{3 / 2}} \exp \left(-c^{2}\right) .
\end{aligned}
$$

Here $n_{s_{i}}, i=1,2$ is the number density of the saturation vapor near each plane interface, calculated from the equation of state using the values of the saturation temperature and corresponding saturation pressure. The number density $n_{r_{i}}, i=1,2$, can be calculated from the impermeability condition on the corresponding interface:

$$
n_{r_{1}}=-2 \sqrt{\pi \frac{T_{1}}{T_{2}}} \int_{c_{y}<0} c_{y} f \mathrm{~d} \mathbf{c}, \quad n_{r_{2}}=2 \sqrt{\pi} \int_{c_{y}>0} c_{y} f \mathrm{~d} \mathbf{c} .
$$

To minimize computational efforts, the $c_{z}$ variable is eliminated by introducing the reduced distribution functions as in [19]. 


\section{Numerical technique}

To solve numerically both linearized and non-linear S-model kinetic equation the same Discrete Velocity Method (DVM) [19] is used. First, the Discrete Velocity Method is applied to split the continuum $(-\infty, \infty)$ molecular velocity space $c_{x}, c_{y}$ in the governing equation (5) into discrete velocity sets $c_{x_{m}}, c_{y_{k}}$ where $m, k=-N_{c}, . .,-1,1,2, . ., N_{c}$. These velocities, $c_{x_{m}}$ and $c_{y_{k}}$, are taken to be the roots of the Hermite polynomial of order $N_{c}$. Then, the set of $2 N_{c}$ kinetic equations, corresponding to $2 N_{c}$ values of discrete velocities, is discretized in space by Finite Difference Method (FDM). In presented here simulations $N_{c}$ is taken to be equal to 20 , so the number of points in the molecular velocity space is equal to 40 in each direction. The grid-independence is checked with finer grid with $N_{c}=25$ points: the difference in all macroscopic quantities was less than $1 \%$.

The spacial derivatives are approximated by the first-order accurate upwind type scheme, see Ref. [19] for more details. The number of uniformly distributed numerical grid points in physical space, $N_{y}$, is equal to 1000 . This number of grid points insures the calculation accuracy of $1 \%$. The convergence criterion for iteration process is employed in following form

$$
\min _{i}\left|q_{i}^{(l+1)}-q_{i}^{(l)}\right| /\left|q_{i}^{(l)}\right| \leq \varepsilon,
$$

where $l$ is the iteration number, $i=1, N_{y} ; \varepsilon=10^{-7}$ is used here.

The described above numerical approach was used to solve the linearized and non-linear S-model kinetic equation for the large range of the rarefaction parameter and for several values of the evaporation coefficients. The obtained results are discussed in Section 6.

\section{Solution of the linearized Navier-Stokes equations with the jump boundary conditions}

The numerical solution of the model kinetic equation provide very accurate information about the evaporation-condensation process. However, from the practical point of view it is interesting to have the simple and easy to use estimation of the vapor behaviors. Below the analytical solution of the linearized Navier-Stokes equations with the energy equation subjected with the temperature and pressure jump boundary conditions is derived.

For one dimensional in the physical space case, considered here, the linearized Navier-Stokes equations, completed with the energy equations read:

$$
\frac{d u_{y}^{\prime}}{d y^{\prime}}=0, \quad \frac{d p^{\prime}}{d y^{\prime}}=0, \quad \frac{d^{2} T^{\prime}}{d y^{\prime 2}}=0 .
$$

To take into account the evaporation-condensation on the upper and down interfaces, equations (37) have to be subjected to the special boundary conditions [9], [20], [21], [10]. We will use here the most complete form of the boundary 
conditions suggested by the authors of Ref. [9] and which relate the temperature and pressure jumps on the liquid-vapor interface, the driving "forces", with the "fluxes", evaporation rate and heat flux through interface. For the down plate this relation between "fluxes" and "forces" reads:

$$
\left[\begin{array}{c}
\left(p_{1}-p^{\prime}\right) / p_{1} \\
\left(T_{1}-T^{\prime}\right) / T_{1}
\end{array}\right]=\left[\begin{array}{ll}
a_{11}^{\prime} & a_{12}^{\prime} \\
a_{21}^{\prime} & a_{22}^{\prime}
\end{array}\right] \times\left[\begin{array}{c}
n_{1} u^{\prime} \\
q^{\prime} /\left(k_{\mathrm{B}} T_{1}\right)
\end{array}\right] .
$$

The heat flux in system $(38)$ reads:

$$
q^{\prime}=-\kappa \frac{d T^{\prime}}{d y^{\prime}}
$$

where $\kappa$ is the thermal conductivity coefficient, which can be calculated as:

$$
\kappa=\frac{\gamma}{\gamma-1} \frac{1}{\operatorname{Pr}} \mathcal{R} \mu
$$

where $\gamma$ is the heat capacity ratio, $\operatorname{Pr}$ is the Prandtl number. For the monatomic gas considered here, $\gamma=5 / 3$ and $\operatorname{Pr}=2 / 3$.

The matrix in Eq. (38) is the inverse Onsager matrix, so it is symmetric and $a_{12}^{\prime}=a_{21}^{\prime}$. For further derivation it is convenient to introduce the reduced form:

$$
a_{i j}=a_{i j}^{\prime} /\left(n_{1} v_{1}\right)
$$

The values of these coefficients were calculated in Refs. [9], [20], [21], [10] for the monatomic gases and for the case of the diffuse reflection of the molecules which do not condense on the surface:

$$
a_{11}=\frac{2 \sqrt{\pi}}{\sigma}-1.4195228, \quad a_{12}=a_{21}=0.446658, \quad a_{22}=1.0422028 .
$$

It is worth to note that only $a_{11}$ coefficient depends on the evaporation coefficient $\sigma$.

In our case of the evaporation-condensation between two parallel condensed surfaces maintained at different temperatures the boundary conditions, Eqs. (38), are write as following:

for the bottom condensed surface $y=0$ :

$$
p^{\prime}=p_{1}\left(1-a_{11} \frac{u^{\prime}}{v_{1}}-a_{12} \frac{q^{\prime}}{v_{1} p_{1}}\right), \quad T^{\prime}=T_{1}\left(1-a_{21} \frac{u^{\prime}}{v_{1}}-a_{22} \frac{q^{\prime}}{v_{1} p_{1}}\right)
$$

for the upper condensed surface $y=1$ :

$$
p^{\prime}=p_{2}\left(1+a_{11} \frac{u^{\prime}}{v_{2}}+a_{12} \frac{q^{\prime}}{v_{2} p_{2}}\right), \quad T^{\prime}=T_{2}\left(1+a_{21} \frac{u^{\prime}}{v_{2}}+a_{22} \frac{q^{\prime}}{v_{2} p_{2}}\right),
$$

Taking into account the conditions of the smallness of the pressure and temperature gradients, Eqs. (11), linearized system of the Navier-Stokes equations 
(37) with the boundary conditions (43), (44) was solved analytically. The analytical dimensionless expressions read:

$$
\begin{gathered}
T(y)=\mathcal{A} y+\mathcal{B}, \\
\mathcal{A}=\frac{a_{12}}{a_{11}} \mathcal{C} X_{P}-\mathcal{C} X_{T}, \quad \mathcal{B}=1-\frac{a_{12}}{2 a_{11}} \mathcal{C} X_{P}-\left(1+\frac{\eta}{2 \delta} \mathcal{M}\right) \mathcal{C} X_{T}, \\
p=1+\frac{1}{2} X_{P}, \\
u=\left(\frac{1}{2 a_{11}}+\frac{a_{22}}{a_{11}} \frac{\eta}{2 \delta}\right) \mathcal{C} X_{P}-\frac{a_{12}}{a_{11}} \frac{\eta}{2 \delta} \mathcal{C} X_{T}, \\
q=-\frac{a_{12}}{a_{11}} \frac{\eta}{2 \delta} \mathcal{C} X_{P}+\frac{\eta}{2 \delta} \mathcal{C} X_{T}, \\
J_{n}=u, \\
\mathcal{M}=\frac{\gamma}{\gamma-1} \frac{1}{P r}, \quad a_{22}-\frac{a_{12}^{2}}{a_{11}}, \quad \mathcal{C}=\left(1+\frac{\eta}{\delta} \mathcal{M}\right)^{-1} .
\end{gathered}
$$

From the analysis of analytical solutions, Eqs. (45) - (49), it is clear that the temperature profile is linear while the evaporation velocity, pressure and heat flux in the gap are constant. The form of the analytical solutions is analogous to the form of Eqs.(18), where the temperature, velocity and heat flux are split into two parts: one part related to the pressure gradient and the second one related to the temperature gradient. In Section 6 both solutions: the analytical expressions, Eqs. (45) - (49), and the numerical solutions of the linearized Smodel equation are compared in their separated (according to the corresponding gradients) form.

It is worth to note that the Onsager-Casimir relation leads here to the equality between the mass flux caused by the temperature drop $u_{T}$ and the thermal flux caused by the pressure drop $q_{P}[15]$ :

$$
u_{T}=q_{P},
$$

see analytical expressions, Eqs. (48), (49).

From the boundary conditions on the down plate, Eqs. (43), we can see that the temperature jump on the liquid-vapor interface depends on both evaporation velocity and heat flux. According to Eqs. (43) the temperature of the gas over an evaporating surface is lower than that of the condensed phase [21]. Because of this temperature drop the "negative temperature gradient" phenomenon is observed when the rate of evaporation (or $X_{P} / X_{T}$ ) is sufficiently large [6], [21]. However, when $X_{P} / X_{T}$ is very small, the mass flow rate is directed from the colder to the hotter condensed phase. This situation was observed in several recent measurements, see [22], [23], [24]. However in all these papers the positive temperature jump the vapor temperature is higher as that of the liquid phase, was observed while the linearized approach predicts the negative one. 


\section{Results and discussion}

We present the results in following manner. First, the results obtained numerically from the linearized S-model kinetic equation and the analytical solution of the linearized Navier-Stokes equations subjected to the jumps boundary conditions are considered in order to show the limits of the applicability of this analytical solution in term of the rarefaction parameter. The cases of complete and non-complete evaporation-condensation are also presented and compared. Then, the linearized and non-linear numerical solutions of the S-model kinetic equation are compared to show the limits of the linearized approach application. Finally, the numerical solutions obtained from the S-model kinetic equation are compared with those giving by the BGK model in linearized and non-linear formulations.

\subsection{Linearized approaches, complete evaporation}

In this Section we will present the results obtained from the numerical solution of the linearized S-model kinetic equation and the analytical solution of the linearized Navier-Stokes equations completed by the pressure and temperature jump boundary conditions. Both solutions are determined only by two parameters: the gas rarefaction $\delta$ and the evaporation-condensation coefficient $\sigma$. Figure 2 shows the variations of the macroscopic vapor parameters: dimensionless number density and temperature in the gap between two condensed phases, obtained from the numerical solution of the linearized S-model equation. The case of the complete evaporation-condensation is considered, $\sigma=1$, for four values of the rarefaction parameter: $\delta=0.1,1,10$ and 100. The macroscopic parameters are separated in two parts: one part due to the pressure gradient (subscript $P$ ) and another one due to the temperature gradient (subcript $T$ ), so we consider here these two gradients to be independent. From Figure 2 we can see that both dimensionless number density and temperature vary quasi linearly in the gap. The profiles of the number density, related to the pressure gradient, $\nu_{P}$, are almost coincide for the values of the rarefaction parameter $\delta=1,10$ and 100, while the profiles of the number density variation due to the temperature gradient, $\nu_{T}$, are different for different values of the rarefaction parameter. The profiles of the dimensionless temperatures, $\tau_{P}$ and $\tau_{T}$, show the "mirror" behavior in the gap: $\tau_{P}$ increases while $\tau_{T}$ decreases from down to upper condensed phases.

The evaporation velocity and the heat flux are constant in the gap, so their average over the gap values for different gas rarefaction are presented in Table 1. It is to note that the gas velocity generated by temperature gradient, $u_{T}$, is oriented in the direction opposite to the evaporation flow generated by the pressure gradient, $u_{P}$. In addition, the values of $u_{T}$ velocity is much lower than that of $u_{P}$ velocity, especially in the case of large value of rarefaction parameter, $\delta=100$. In this regime, the evaporation rate is engendered essentially by the pressure gradient. The evaporation velocities, $u_{P}$ and $\left|u_{T}\right|$, decrease with rarefaction parameter increases. 


\begin{tabular}{|c|c|c|c|c|c|c|}
\hline \multirow{2}{*}{} & \multicolumn{3}{|c|}{ S-model } & \multicolumn{3}{c|}{ Navier-Stokes } \\
\cline { 2 - 7 } & $\delta=1$ & 10 & 100 & 1 & 10 & 100 \\
\hline$u_{P}$ & 0.2578 & 0.2408 & 0.2356 & 0.2526 & 0.2406 & 0.2361 \\
\hline$u_{T}$ & -0.0911 & -0.0290 & -0.0038 & -0.0862 & -0.0290 & -0.0038 \\
\hline$q_{T}$ & 0.4327 & 0.1385 & 0.0181 & 0.4114 & 0.1383 & 0.0181 \\
\hline
\end{tabular}

Table 1: Macroscopic parameters obtained from the numerical solution of the linearized Smodel kinetic equation and the analytical solution of the Navier-Stokes equations with the jump boundary conditions for different values of the rarefaction parameter $\delta$ and for complete evaporation, $\sigma=1$.

It can be seen from Table 1 that the heat flux generated by the temperature gradient, $q_{T}$, is positive and directed from the hot bottom plate to the cold upper plate, while the heat flux generated by the pressure gradient, $q_{P}$, has the opposite sign and is much smaller in absolute value. The values of the heat flux related to the pressure gradient, $q_{P}$, are not provided in Table 1, because their values can be found from the Onsager-Casimir reciprocity relations $q_{P}=u_{T}$, see Eq. (52). Both heat fluxes, $\left|q_{P}\right|$ and $q_{T}$, decrease with $\delta$ increasing, see Table 1.

By analyzing the results provided in Table 1 one can conclude that the difference between the analytical solution of the linearized Navier-Stokes equations subjected by the jump boundary conditions and the numerical solution of the S-model kinetic equation is very small. The difference between both solutions for $\delta=10$ is of the order of $0.1 \%$. For larger value of the rarefaction parameter, $\delta=1$, this difference keeps surprisingly small, around $5 \%$. Therefore, analytical expressions, Eqs. (45) - (49), can be used for estimation of the evaporation rate with a good accuracy up to $\delta=10$.

\subsection{Linearized approaches, non-complete evaporation}

Two approaches, the numerical solution of the S-model kinetic equation and the analytical solution of the Navier-Stokes equations subjected to the jump boundary conditions, are analyzed now for the case of non-complete evaporation. The cases of $\sigma=0.2,0.5,0.8$ and 1 are considered. Figure 3 shows the influence of the non-complete evaporation-condensation on the macroscopic profiles of the number density and temperature, obtained from the numerical solution of the linearized S-model kinetic equation. The results for four values of the evaporation coefficient, $\sigma=0.2,0.5,0.8$ and 1, are presented on Fig. 3 for rarefaction parameter $\delta=10$. It is worth to note that the profiles of the number density and temperature, caused by the temperature gradient, $\nu_{T}$ and $\tau_{T}$, are independent from the evaporation coefficient, so they depend only on the temperature gradient between the condensed surfaces.

As it was mentioned above, the evaporation velocity and the heat flux are constant in the gap, so their averaged over the gap values, obtained from the numerical solution of the linearized S-model kinetic equation and the analytical solution of the linearized Navier-Stokes equations, Eqs. (45) - (49), are presented in Table 2 for the case of non-complete evaporation, $\sigma=0.5$, and different 
values of the rarefaction parameter $\delta$. Comparing the values of the evaporation velocity, provided in Tables 1 and 2 one can conclude that the decrease in the evaporation coefficient decreases the absolute value of both evaporation velocities. The heat flux caused by temperature gradient, $q_{T}$, remains practically the same with $\sigma$ decreases, while the values of the heat flux caused by pressure gradient, $q_{P}=u_{T}$, see Table 2 , decrease in approximately two times when the evaporation coefficient reduces from 1 to 0.5 , see also Table 1 . This behavior of the heat flux is in agreement with the temperature behaviors, presented on Fig. 3: temperature $\tau_{T}$, due to the temperature gradient, remains the same when the evaporation coefficient changes. It is worth to note that as for the case of the complete evaporation, the agreement between numerical solution of the linearized S-model kinetic equation and the analytical solution of the NavierStokes equation is very good: the difference between both solutions for $\delta=10$ is of the order of $1 \%$.

\begin{tabular}{|c|c|c|c|c|c|c|}
\hline \multirow{2}{*}{} & \multicolumn{3}{|c|}{ S-model } & \multicolumn{3}{c|}{ Navier-Stokes } \\
\cline { 2 - 7 } & $\delta=1$ & 10 & 100 & 1 & 10 & 100 \\
\hline$u_{P}$ & 0.0912 & 0.0891 & 0.0888 & 0.0906 & 0.0890 & 0.0883 \\
\hline$u_{T}$ & -0.0322 & -0.0108 & -0.0015 & -0.0309 & -0.0107 & -0.0014 \\
\hline$q_{T}$ & 0.4119 & 0.1363 & 0.0181 & 0.3926 & 0.1361 & 0.0181 \\
\hline
\end{tabular}

Table 2: Macroscopic parameters obtained from the numerical solution of the linearized Smodel kinetic equation and the analytical solution of the Navier-Stokes equations with the jump boundary conditions for different values of the rarefaction parameter $\delta$ and for noncomplete evaporation, $\sigma=0.5$.

\subsection{Comparison of the BGK and S-model numerical solutions}

As it was underlined in the Introduction, the S-model is chosen here because it provides the correct Prandtl number, equal to $2 / 3$ for monatomic gases, contrary to the BGK model, for which the Prandtl number is equal to 1. In this Section both linear and non-linear BGK and S-model solutions are compared to see the influence of the applied models on the macroscopic profiles.

First, the numerical solutions of the linearized BGK and S-model equations are shown on Fig. 4 for the case of weak evaporation and condensation, $T_{1} / T_{2}=$ $1.001, p_{1} / p_{2}=1.01$ and for the large value of the rarefaction parameter, $\delta=$ 250. It can be seen from Fig. 4 that both linearized solutions are in good agreement, except for the heat flux profiles, as expected. However, the heat flux profiles obtained by the linearized S-model equation and linearized NavierStokes equations coincide, because both models have the same Prandtl number.

Let us compare now the solutions of the non-linear BGK and S-model equations. Tables 3 and 4 provide the numerical values of the dimensionless evaporation rate, Eq. (9), $J_{n}^{\prime} /\left(n_{2} v_{2}\right)$ and energy flux, Eq. (10), $J_{E}^{\prime} /\left(p_{2} v_{2}\right)$. Both quantities are obtained by integration of the kinetic equation, Eq. (5), and show the conservation of momentum and energy. Both quantities are independent from the type of the kinetic model and show the reliability of applied numerical methods to obtain the numerical solutions. The temperature and pressure 
ratios were chosen according to Ref. [8]. As it is clear from Tables 3 and 4 very good agreement was found between the presented here results (BGK and S-model) and the BGK results from Ref. [8] for the dimensionless evaporation rate, $J_{n}$ and energy flux $J_{E}$. The difference between three solutions is of the order of $1 \%$.

The profiles of the macroscopic parameters, obtained from the numerical solution of the BGK and S-model equations are however slightly different, especially, as it was expected, the profiles of heat flux, which was also observed when comparing the linearized versions of both models. Figure 5 illustrates this difference in the macroscopic profiles of the evaporation velocity and heat flux in the gap between condensed phases. The comparison with the measurements are needed to conclude definitively about the advantages of one or other models.

\begin{tabular}{|c|c|c|c|c|c|c|}
\hline \multirow{3}{*}{$\delta$} & \multicolumn{3}{|c|}{$T_{H} / T_{C}=1.01$} & \multicolumn{3}{c|}{1.2} \\
\cline { 2 - 7 } & \multicolumn{3}{|c}{$P_{H} / P_{C}=1.02$} & \multicolumn{3}{c|}{2} \\
\cline { 2 - 7 } & BGK & S-mod & BGK [8] & BGK & S-mod & BGK [8] \\
\hline 0.090 & 0.00421 & 0.00420 & 0.00421 & 0.22936 & 0.22925 & 0.22891 \\
0.903 & 0.00427 & 0.00422 & 0.00429 & 0.22151 & 0.22103 & 0.22109 \\
9.027 & 0.00454 & 0.00450 & 0.00457 & 0.22025 & 0.22006 & 0.22030 \\
\hline
\end{tabular}

Table 3: Dimensionless evaporation rate $J_{n}$

\begin{tabular}{|c|c|c|c|c|c|c|}
\hline \multirow{3}{*}{$\delta$} & \multicolumn{3}{|c|}{$T_{H} / T_{C}=1.01$} & \multicolumn{3}{c|}{1.2} \\
\cline { 2 - 7 } & \multicolumn{3}{|c}{$P_{H} / P_{C}=1.02$} & \multicolumn{3}{c|}{2} \\
\cline { 2 - 7 } & BGK & S-mod & BGK [8] & BGK & S-mod & BGK [8] \\
\hline 0.090 & 0.01395 & 0.01397 & 0.01391 & 0.66267 & 0.66293 & 0.66141 \\
0.903 & 0.01308 & 0.01320 & 0.01296 & 0.63507 & 0.63633 & 0.63287 \\
9.027 & 0.01206 & 0.01217 & 0.01201 & 0.62106 & 0.62161 & 0.62105 \\
\hline
\end{tabular}

Table 4: Dimensionless energy flux $J_{E}$

\subsection{Evaporation-condensation of Argon}

In this Section we establish the applicability domain of the linearized S-model kinetic equation and of the analytical expressions (45) - (49). Contrarily to the linearized equation, to solve the non-linear S-model equation we need to fix the pressure and temperature ratios between two condensed surfaces. Usually the authors [8] consider the variations of the saturation temperature and pressure independently, but here we consider more realistic case.

The S-model kinetic equation describes the behavior of the monatomic gases. Therefore, we fixed three temperature ratios, $T_{1} / T_{2}=1.001,1.01$ and 1.05 and estimated the corresponding saturation pressures for some monatomic gases, taking into account the Clayperon-Clausius equation [25]. The fixed temperature ratios of two condensed phases, $T_{1} / T_{2}=1.001,1.01$ and 1.05 , lead to corresponding pressure ratios $p_{1} / p_{2}=1.01,1.1,1.55$ for Krypton, starting with 
temperature $120 K$, to pressure ratios $p_{1} / p_{2}=1.01,1.1,1.57$ for Argon, if we start with the temperature $T_{2}=84 K$ and to pressure ratios $p_{1} / p_{2}=1.004$, 1.04, 1.2 for Helium. Some approximative tendency is so deduced: the increase of saturation temperature in $1 \%$ causes the increase of saturation pressure in approximately $10 \%$. Therefore, the three sets of saturation parameters are chosen to solve numerically the non-linear S-model kinetic equation: $T_{1} / T_{2}=1.001$, 1.01, 1.05 and $p_{1} / p_{2}=1.01,1.1,1.5$ (see also two first lines in Table 5) to analyze the eventual limits of application for the linearized models.

\begin{tabular}{|c|c|c|c|c|c|c|c|c|c|}
\hline \multirow{2}{*}{$\delta$} & \multicolumn{3}{|c|}{$T_{1} / T_{2}=1.001$} & \multicolumn{3}{c|}{1.01} & \multicolumn{3}{c|}{1.05} \\
\cline { 2 - 10 } & \multicolumn{3}{|c|}{$p_{1} / p_{2}=1.01$} & \multicolumn{3}{c|}{1.1} & \multicolumn{3}{c|}{1.5} \\
\hline & Lin S & Lin NS & Non-l S & Lin S & Lin NS & Non-l S & Lin S & Lin NS & Non-l S \\
\hline 1 & 0.00250 & 0.00245 & 0.00248 & 0.02599 & 0.02545 & 0.02469 & 0.15235 & 0.14842 & 0.12092 \\
\hline 10 & 0.00239 & 0.00238 & 0.00237 & 0.02486 & 0.02480 & 0.02367 & 0.14573 & 0.14473 & 0.11684 \\
\hline 100 & 0.00236 & 0.00236 & 0.00235 & 0.02458 & 0.02452 & 0.02344 & 0.14410 & 0.14310 & 0.11654 \\
\hline
\end{tabular}

Table 5: Dimensionless evaporation rate $J_{n}$ obtained by: the numerical solution of the linearized S-model kinetic equation (Lin S), the analytical expression Eq.(50), (Lin NS), and the numerical solution of the non-linear S-model kinetic equation (Non-l S).

Table 5 provides the values of dimensionless evaporation rate, $J_{n}$, obtained by: numerical solution of the linearized S-model kinetic equation, analytical expression, Eq.(50), and numerical solution of the non-linear S-model kinetic equation. By comparing the results obtained from these three approaches it is clear that for two first pairs of saturation temperature/pressure values all the approaches gives very similar results. The difference between two linearized approaches does not exceed $0.2 \%$. It is to note that the values of analytical solution of the linearized Navier-Stokes equations are provided in Table 5 also for $\delta=1$, even if theoretically this approach is not valid for $\delta<10$. But for given conditions the good agreement was found between all three approaches also for $\delta=1$. However, the difference between numerical solutions of the linearized and non-linear S-model kinetic equations becomes of the order of $4.6 \%$ for the temperature ratio 1.01 and this difference increases up to $20 \%$, when the saturation temperature ratio increases up to 1.05. In the same time the agreement between two linearized approaches remains very good, of the order of $1 \%$ even for relatively large temperature ratio of 1.05 . From the presented analysis, we can conclude that the linearized approaches work well for the real monatomic gases, i.e. taking into account their respective Clausius-Clayperon relations, only up to very small temperature ratio of the order of $1 \%$, and therefore for the larger temperature ratios the non-linear approaches have to be applied.

The general tendency of the evaporation rate behavior can be derived by analyzing the results from Table 5: the evaporation rate decreases slightly when the rarefaction parameter increases for the fixed saturation temperature/pressure ratios. The evaporation rate increases more than in 10 times when the saturation temperature ratio increases in 10 times, this tendency of the proportionality 
remains up to temperature ratio equal to 1.05 .

The numerical solutions of the linearized and non-linear S-model equations and the analytical solution of the linearized Navier-Stokes equations subjected to the pressure and temperature jumps boundary conditions, Eqs. (45) - (49), are presented on Figs. 6 - 9 for two values of the rarefaction parameter $\delta=10$ and 100 , for the case of the complete evaporation, $\sigma=1$, and for three values of the saturation temperature/pressure ratios, presented in Table 5. The macroscopic profiles of the temperature, pressure, evaporation velocity and heat flux in the gap are provided.

It is seen from these Figures that all the temperature and pressure profiles for two temperature ratios $T_{1} / T_{2}=1.001$ and 1.01 practically coincide for two values of the rarefaction parameter, $\delta=10$ and 100, except, the case, shown on Fig. 8(c), where the temperature profile becomes non-linear, in agreement with the findings of Ref. [8]. In this case, the maximum of the temperature/pressure ratio is smaller than the rarefaction parameter and the linearized solutions do not coincide with the non-linear one, even if the deviations of the macroscopic parameters from their equilibrium state is small enough and normally the linearized theory should work.

With further saturation temperature increasing, up to $T_{1} / T_{2}=1.05$, the temperature profiles, provided by the non-linear S-model equation, start to deviate from the linear ones for both value of $\delta$, while the pressure profiles keep their shape and only absolute values of pressure change. The profiles of the evaporation velocity and of the heat flux differ from those provided by the linearized approaches even for small temperature ratio. For larger temperature ratios, $T_{1} / T_{2}=1.01$ and 1.05 , the behaviors of the linearized and nonlinear models differ not only quantitatively, but also qualitatively. Especially it is clearly seen near the liquid boundaries for heat flux distributions, Figs. 7 and 9 and for temperature distributions, Figs. 6 and 8. The pressure profiles, obtained from all models and for all considered rarefaction levels, have the same qualitative behavior: fully coincide for $T_{1} / T_{2}=1.001$ and have a difference in $2 \%$ for $T_{1} / T_{2}=1.05$.

It is to note that the analytical solution of the linearized Navier-Stokes equations, even subjected with the temperature and pressure jump boundary conditions, cannot provide the correct profiles of the macroscopic parameters inside thin layer near condensed phases, the Knudsen layer, see the temperature, evaporation velocity and heat flux profiles. Even for very small pressure and temperature ratios, $1 \%$ and $0.1 \%$, respectively, the profiles given by the analytical solutions are different, see Figs. 6 - 9 (a) and (b). However, the analytical results provide acceptable accuracy of the macroscopic parameters outside of the Knudsen layer up to temperature ratio $T_{1} / T_{2}=1.01$ and can be used for rough estimations.

An interesting remark can be done concerning the limits of validity of the linearized approach: the consideration of the evaporation and condensation phenomena reduces considerably this limit. It was found in [26] that the heat flux between two parallel solid plates, obtained from the numerical solution of the non-linear S-model equation, still keeps its linear behavior up to temperature 
ratio equal to $T_{1} / T_{2} \approx 3$, which is much larger compared to the limit found in present analysis.

It is worth to note that the "inversion of the temperature gradient", mentioned for the first time in Refs. [6], [7], is observed in the gap for all considered here cases. With the temperature gradient inversion the temperature near the hotter condensed surface becomes lower than that near the colder one. This phenomenon is obtained in numerical simulations, when the ratio between the pressure and temperature differences, $X_{P} / X_{T}$ becomes larger than 4.772337 [21]. Here this criterion is satisfied for all calculated cases, so the vapor temperature is larger near the colder condensed surface, see Figs. 6 and 8.

\section{Conclusion}

The linearized and non-linear S-model equations are solved for different values of the rarefaction parameters and several saturation pressures and temperatures ratios. The case of the non-complete evaporation-condensation is also considered. The comparison between the BGK and S-model numerical solutions shows the difference only in the heat flux profiles while the other macroscopic parameters have not beed impacted by the model changing. The analytical solution of the linearized Navier-Stokes equations completed by the pressure and temperature jumps boundary conditions is obtained. The numerical solutions of the linearized and non-linear kinetic equations are compared together and with the analytical solutions of the continuum equations. It was found that the analytical solutions of the Navier-Stokes equations provide the excellent agreement with the numerical solutions of the linearized BGK and S-model kinetic equations up to rarefaction parameter equal to 10, in the case of complete and non-complete evaporation-condensation. The case of the real relations between saturation temperature/pressure for some monatomic gases are considered and it was found that the linearized approaches give satisfactory results only for the temperature deviation between condensed phases of the order of $1 \%$, but for the larger saturation temperature ratio the non-linear approach has to be applied.

\section{Acknowledgements}

The research work of one of the authors (A. Polikarpov) was funded by RFBR according to the research project no. 18-31-00194. A.Polikarpov also thanks the Ministry of Education and Science of the Russian Federation Agreement no. 02.A03.21.0006 for travel grant. Irina Graur thanks Prof. Aldo Frezzotti for the helpful discussions.

[1] Y. Sone, Kinetic Theory and Fluid Mechanics, Birkhäuser, Boston, 2002.

[2] R. Meland, Molecular dynamics simulation of the inverted temperature gradient phenomenon, Physic of Fluids 15 (10) (2003) 3244-3247.

[3] H. Hertz, Uber die verdungstung der flussigkeiten, inbesondere des quecksilbers, im luftleeren raume, Ann. Phys. Chem. 17 (1882) 177-200. 
[4] M. Knudsen, Die maximale verdampfungsgeschwindigkeit des que-cksilbers, Ann. Phys. Chem. 47 (1915) 697-708.

[5] A. Schrage, A Theoretical Study of Interphase Mass Transfer, Columbia University Press, New York, 1953.

[6] Y.-P. Pao, Application of kinetic theory to the problem of evaporation and condensation, Phys. of Fluids 14 (2) (1971) 306-312.

[7] Y.-P. Pao, Temperature and density jumps in the kinetic theory of gases and vapors, Phys. Fluids 14 (7) (1971) 1340-1346.

[8] K. Aoki, N. Masukawa, Gas flows caused by evaporation and condensation on two parallel condensed phases and the negative temperature gradient: numerical analysis by using a nonlinear kinetic equation, Phys. Fluids 6 (3) (1994) 1379-1395.

[9] J. W. Cipolla Jr, H. Lang, S. K. Loyalka, Kinetic theory of condensation and evaporation. II, J. Chem. Phys. 61 (1974) 69-77.

[10] Y. Onishi, Y. Sone, Kinetic theory of slightly strong evaporation and condensation - hydrodynamic equation and slip boundary condition for finite reynolds numberevaporation and condensation - hydrodynamic equation and slip boundary condition, J. Phys. Soc. Japan 47 (5) (1979) 1676-1685.

[11] H. Struchtrup, A. Beckmann, A. S. Rana, A. Frezzotti, Evaporation boundary conditions for the r13 equations of rarefied gas dynamics, Physics of Fluids 29 (9) (2017) 092004. arXiv:https://doi.org/10.1063/1.4989570, doi:10.1063/1.4989570.

URL https://doi.org/10.1063/1.4989570

[12] E. M. Shakhov, Generalization of the Krook kinetic relaxation equation, Fluid Dyn. 3 (5) (1968) 95-96.

[13] L. Landau, E. Lifshitz, Statistical physics, Vol. 5 of Course of theoretical physics, Pergamon Press, 1969.

[14] M. N. Kogan, Rarefied gas dynamics, Plenum Press New York, 1969.

[15] F. Sharipov, Onsager-casimir reciprocity relations for open gaseous systems at arbitrary rarefaction. II Application of the theory for single gas, Physica A 203 (1994) 457-485.

[16] C. K. Chu, Kinetic-theoretic description of the formation of a shock wave, Phys. of Fluids 12 (8) (1965) 12-22.

[17] J. E. Broadwell, Shock structure in a simple discrete velocity gas, Physics of fluids 7 (8) (1964) 1243-1247. 
[18] F. Sharipov, Onsager-casimir reciprocity relations for open gaseous systems at arbitrary rarefaction. I General theory for single gas, Physica A 203 (1994) 437-456.

[19] I. A. Graur, A. Polikarpov, Comparison of different kinetic models for the heat transfer problem, Heat and Mass Transfer 46 (2009) 237-244.

[20] H. Lang, Evaporation and condensation for general gas-liquid surface scattering, J. Chem. Phys. 62 (3) (1975) 858-863.

[21] Y. Sone, Y. Onishi, Kinetic theory of evaporation and condensation - hydrodynamic equation and slip boundary condition, J. Phys. Soc. Japan 44 (1978) 1981-1994.

[22] C. A. Ward, D. Stanga, Interfacial conditions during evaporation or condensation of water, Phys. Rev. E 64 (2001) 051509. doi:10.1103/PhysRevE.64.051509.

URL https://link.aps.org/doi/10.1103/PhysRevE.64.051509

[23] V. K. Badam, V. Kumar, F. Durst, K. Danov, Experimental and theoretical invesigations on interfacial temerature jumps during evaporation, Experimental Thermal and Fluid Science 32 (2007) 276-292.

[24] M. A. Kazemi, D. S. Nobes, J. A. Elliott, Experimental and numerical study of the evaporation of water at low pressures, Langmuir 33 (18) (2017) 4578-4591.

[25] L. D. Landau, E. M. Lifshitz, Fluid Mechanics, Pergamon, New York, 1989.

[26] V. G. Chernyak, A. Polikarpov, Non-linear effects in gases in couette problem, Journal of Experimental and Theoretical Physics 110 (1) (2010) 147156. 


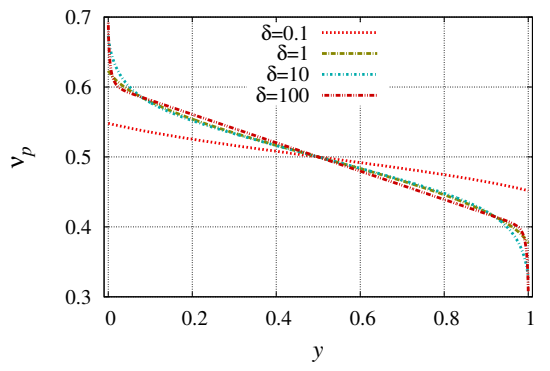

(a)

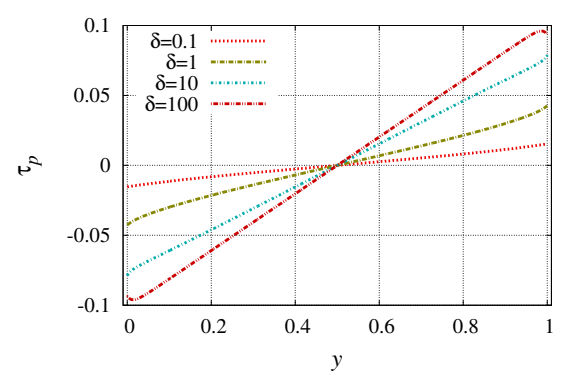

(c)

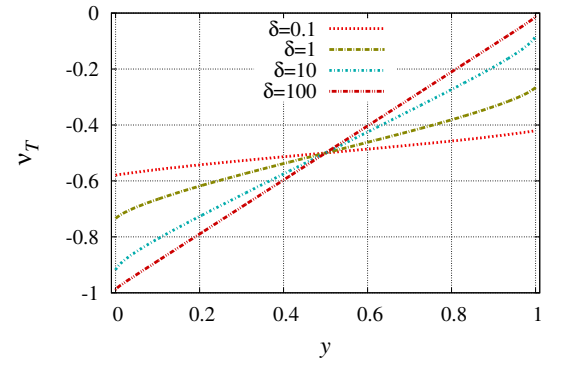

(b)

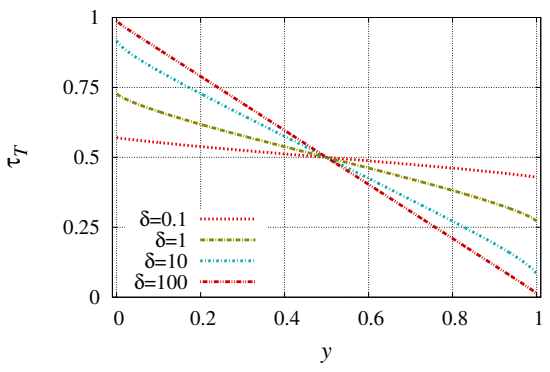

(d)

Figure 2: Numerical solution of the linearized S-model equation: the variations of macroscopic quantities, dimensionless number density and temperature, both separated in two parts: due to the pressure gradient (with superscript $P$, (a) and (c)) and due to the temperature gradient (with superscript $T$, (b) and (c)) in the gap between two condensed phases for different values of the rarefaction parameter $\delta$ and for the case of the complete evaporation, $\sigma=1$. 


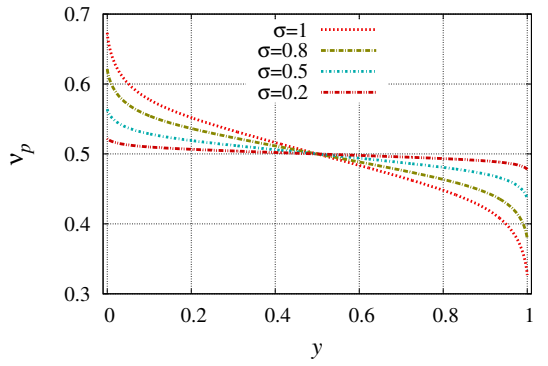

(a)

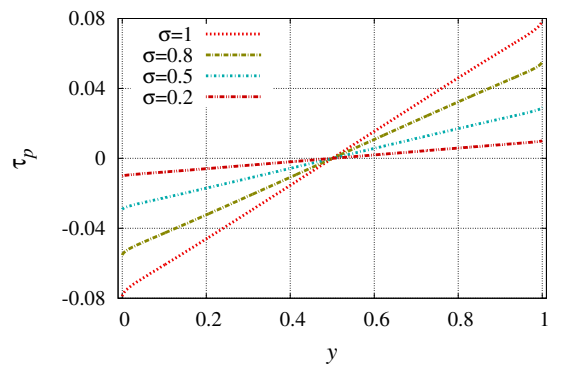

(c)

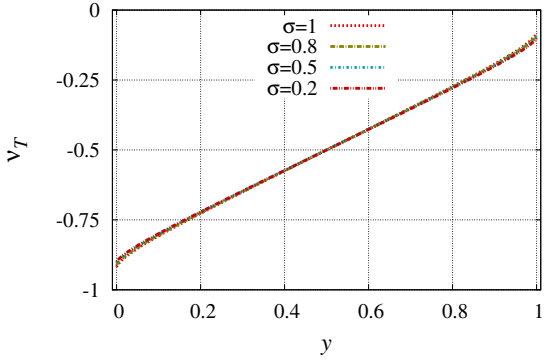

(b)

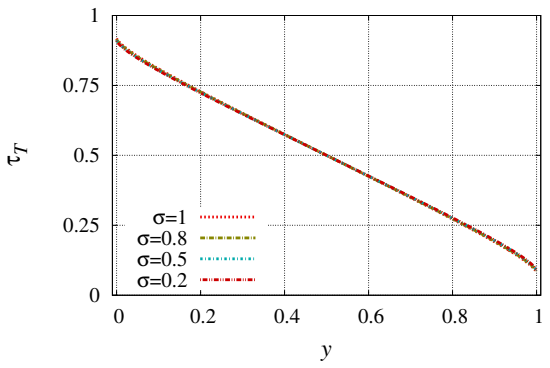

(d)

Figure 3: Numerical solution of the linearized S-model equation: the variations of macroscopic quantities, dimensionless number density and temperature, both separated in two parts: due to the pressure gradient (with superscript $P,(\mathrm{a})$ and (c)) and due to the temperature gradient (with superscript $T$, (b) and (c)) in the gap between two condensed phases for different values of the evaporation coefficient and for the rarefaction parameter $\delta=10$. 


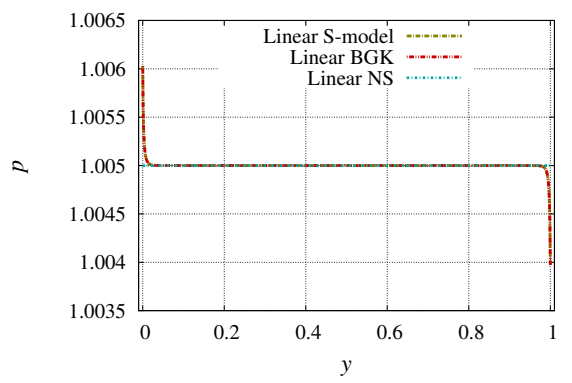

(a)

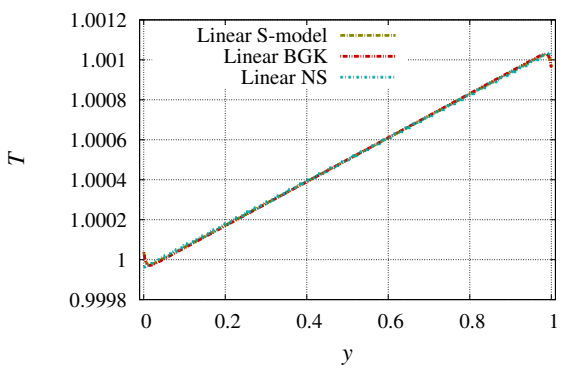

(c)

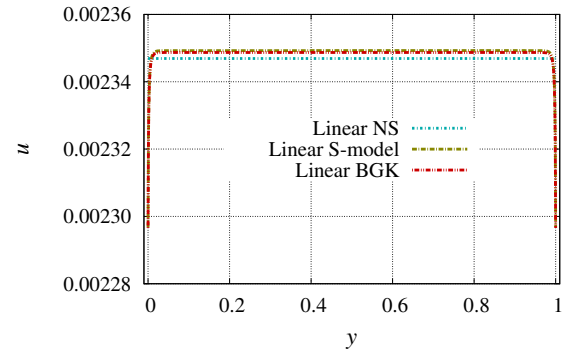

(b)

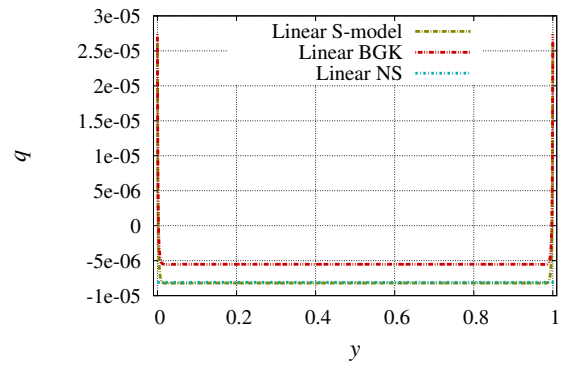

(d)

Figure 4: Temperature (a) and pressure (b), obtained from the numerical solution of linearized BGK and S-model equations, and non-linear S-model equation, $T_{1} / T_{2}=1.001, p_{1} / p_{2}=1.01$, $\delta=250$.

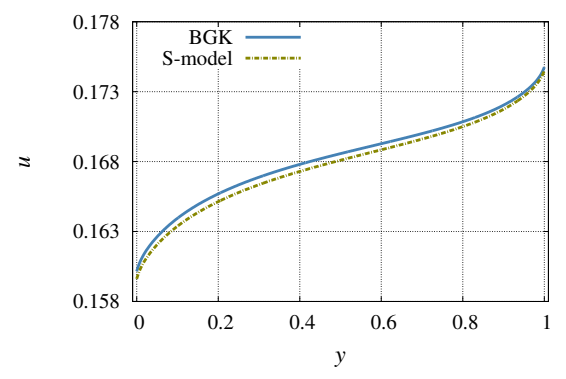

(a)

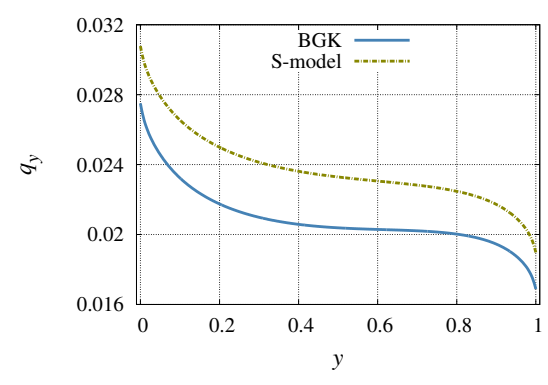

(b)

Figure 5: Evaporation velocity (a) and heat flux (b), obtained from the numerical solution of non-linear BGK and S-model equations, $T_{1} / T_{2}=1.2, p_{1} / p_{2}=2, \delta=0.903$. 


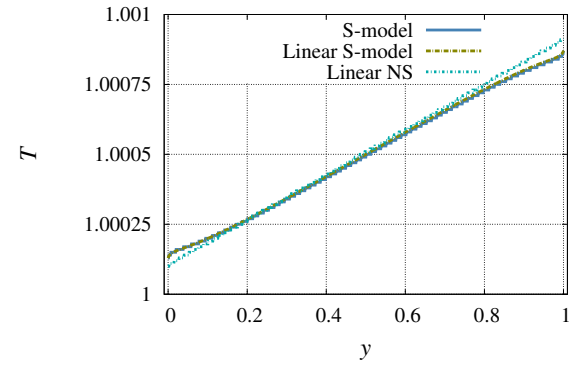

(a) $T_{1} / T_{2}=1.001, p_{1} / p_{2}=1.01$

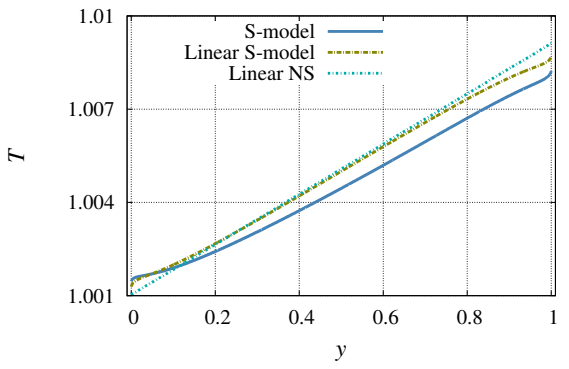

(c) $T_{1} / T_{2}=1.01, p_{1} / p_{2}=1.1$

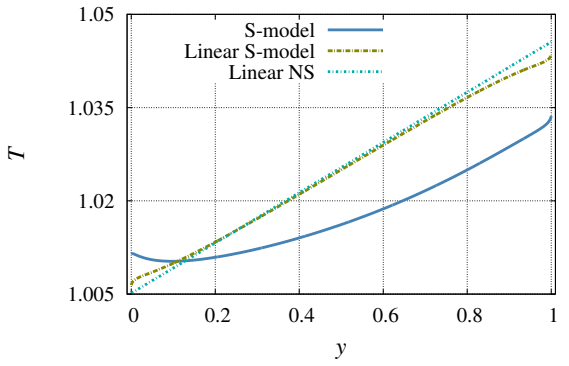

(e) $T_{1} / T_{2}=1.05, p_{1} / p_{2}=1.5$
2

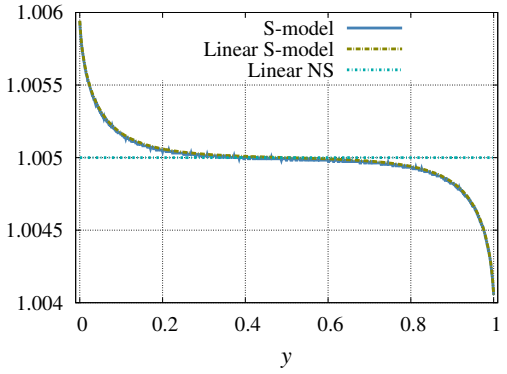

(b) $T_{1} / T_{2}=1.001, p_{1} / p_{2}=1.01$

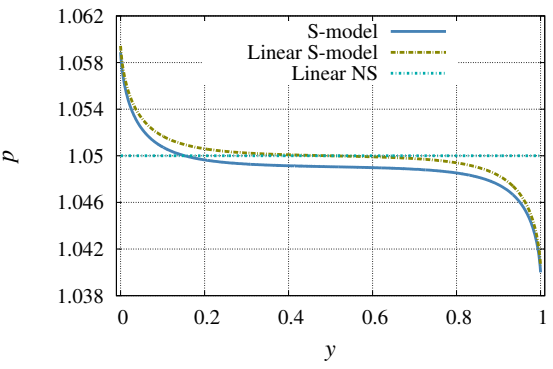

(d) $T_{1} / T_{2}=1.01, p_{1} / p_{2}=1.1$

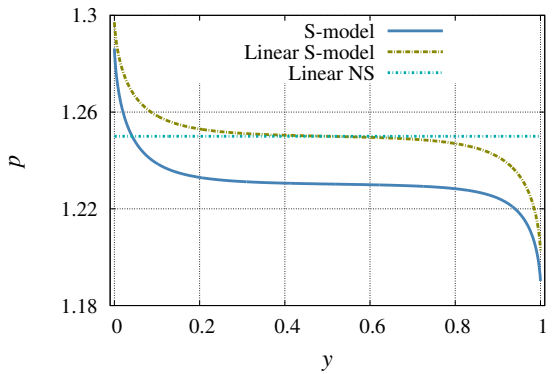

(f) $T_{1} / T_{2}=1.05, p_{1} / p_{2}=1.5$

Figure 6: Comparison of three results for $\delta=10$ and complete evaporation, $\sigma=1$ : the numerical solution of the linearized S-model kinetic equation, the analytical solution of the linearized Navier-Stokes equations with the temperature and pressure jumps boundary conditions and the numerical solution of the non-linear S-model kinetic equation. The temperature and pressure profiles in the gap are compared for three sets of saturation temperature and pressure ratios: (a), (b) $T_{1} / T_{2}=1.001, p_{1} / p_{2}=1.01$, (c), (d) $T_{1} / T_{2}=1.01, p_{1} / p_{2}=1.1$; (e), (f) $T_{1} / T_{2}=1.05, p_{1} / p_{2}=1.5$. 


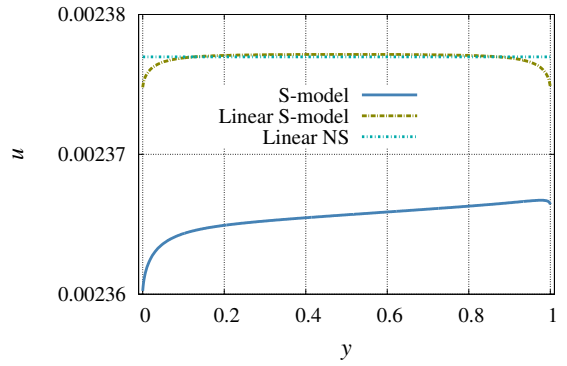

(a) $T_{1} / T_{2}=1.001, p_{1} / p_{2}=1.01$

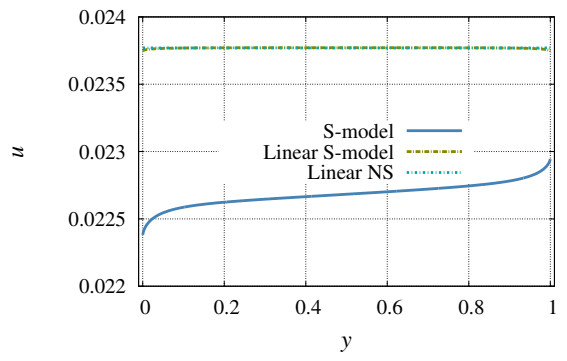

(c) $T_{1} / T_{2}=1.01, p_{1} / p_{2}=1.1$

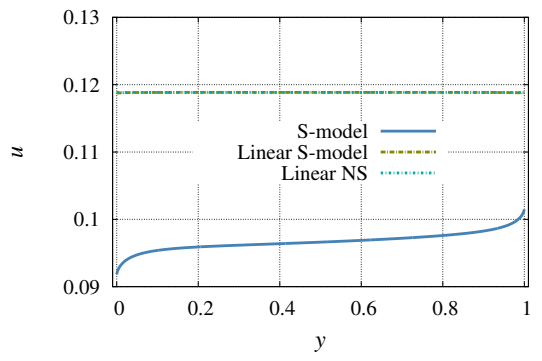

(e) $T_{1} / T_{2}=1.05, p_{1} / p_{2}=1.5$

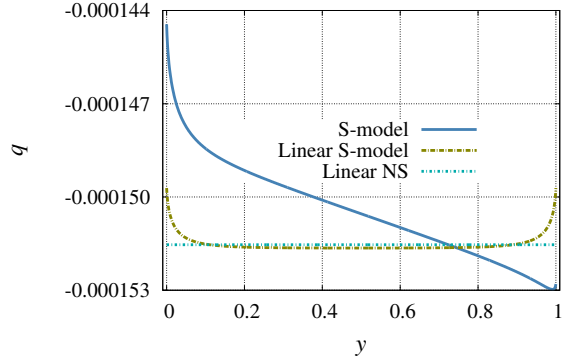

(b) $T_{1} / T_{2}=1.001, p_{1} / p_{2}=1.01$

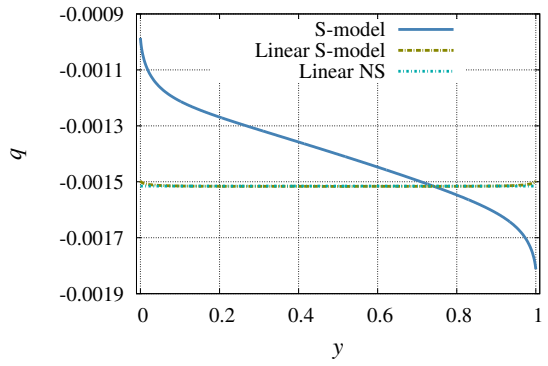

(d) $T_{1} / T_{2}=1.01, p_{1} / p_{2}=1.1$

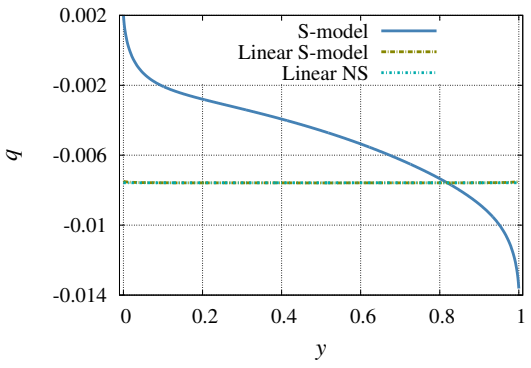

(f) $T_{1} / T_{2}=1.05, p_{1} / p_{2}=1.5$

Figure 7: Comparison of three results for $\delta=10$ and complete evaporation $\sigma=1$ : the numerical solution of the linearized S-model kinetic equation, the analytical solution of the linearized Navier-Stokes equations with the temperature and pressure jumps boundary conditions and the numerical solution of the non-linear S-model kinetic equation. The evaporation velocity and the heat flux profiles in the gap are compared for three sets of saturation temperature and pressure ratios: (a), (b) $T_{1} / T_{2}=1.001, p_{1} / p_{2}=1.01$, (c), (d) $T_{1} / T_{2}=1.01, p_{1} / p_{2}=1.1$; (e), (f) $T_{1} / T_{2}=1.05, p_{1} / p_{2}=1.5$. 


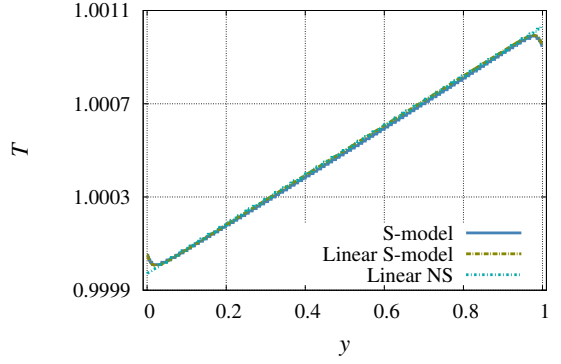

(a) $T_{1} / T_{2}=1.001, p_{1} / p_{2}=1.01$

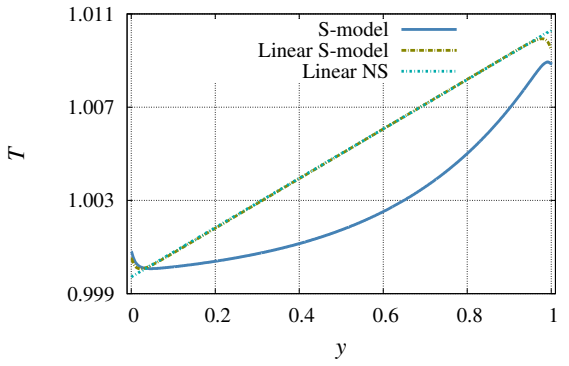

(c) $T_{1} / T_{2}=1.01, p_{1} / p_{2}=1.1$

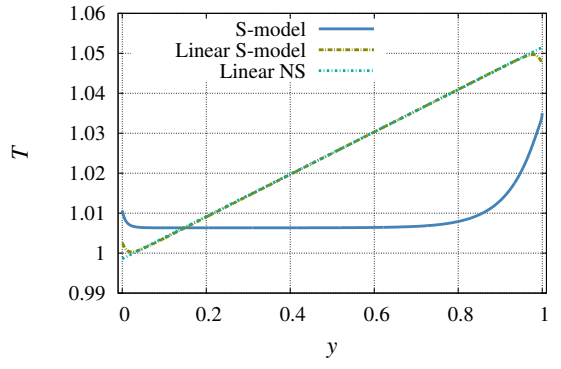

(e) $T_{1} / T_{2}=1.05, p_{1} / p_{2}=1.5$
2

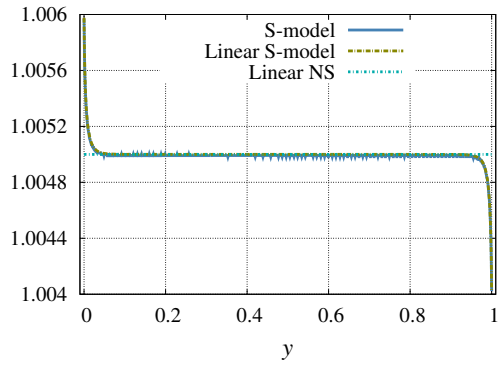

(b) $T_{1} / T_{2}=1.001, p_{1} / p_{2}=1.01$

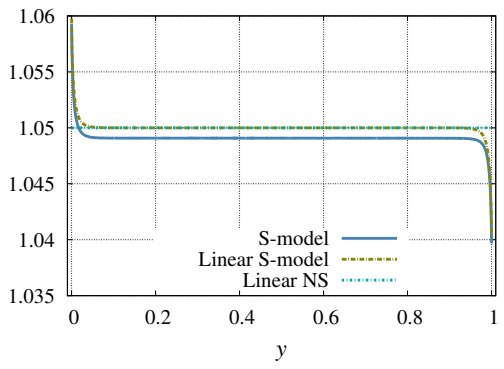

(d) $T_{1} / T_{2}=1.01, p_{1} / p_{2}=1.1$

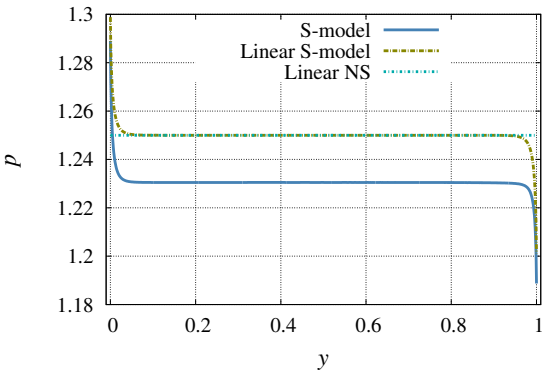

(f) $T_{1} / T_{2}=1.05, p_{1} / p_{2}=1.5$

Figure 8: Comparison of three results for $\delta=100$ and complete evaporation $\sigma=1$ : the numerical solution of the linearized S-model kinetic equation, the analytical solution of the linearized Navier-Stokes equations with the temperature and pressure jumps boundary conditions and the numerical solution of the non-linear S-model kinetic equation. The temperature and pressure profiles in the gap are compared for three sets of saturation temperature and pressure ratios: (a), (b) $T_{1} / T_{2}=1.001, p_{1} / p_{2}=1.01$, (c), (d) $T_{1} / T_{2}=1.01, p_{1} / p_{2}=1.1$; (e), (f) $T_{1} / T_{2}=1.05, p_{1} / p_{2}=1.5$. 


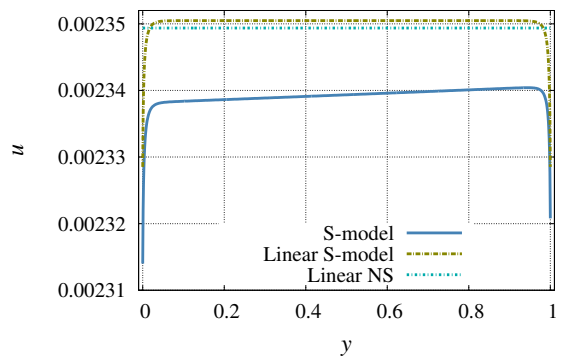

(a) $T_{1} / T_{2}=1.001, p_{1} / p_{2}=1.01$

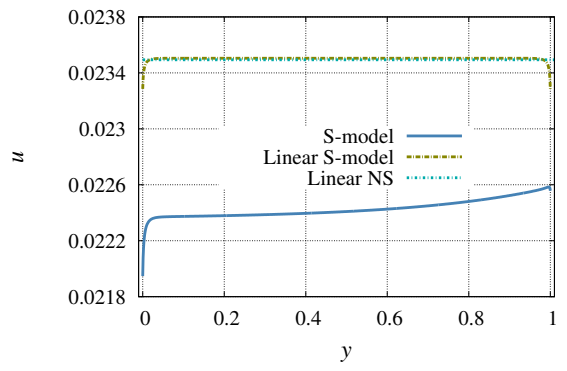

(c) $T_{1} / T_{2}=1.01, p_{1} / p_{2}=1.1$

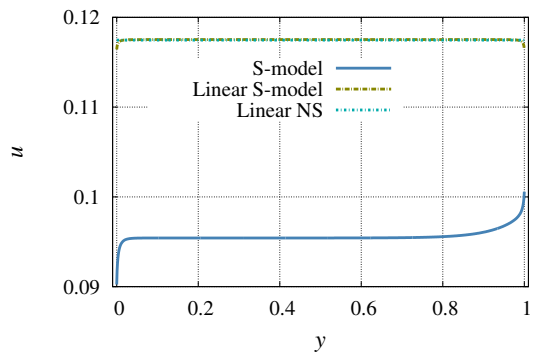

(e) $T_{1} / T_{2}=1.05, p_{1} / p_{2}=1.5$

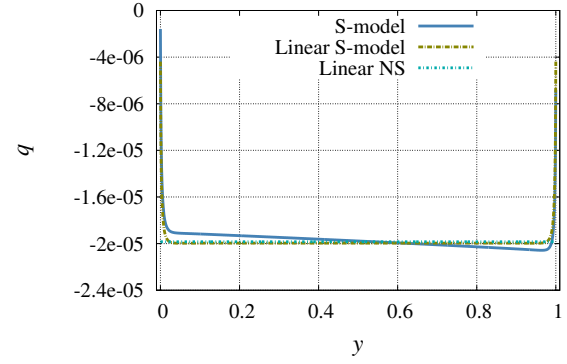

(b) $T_{1} / T_{2}=1.001, p_{1} / p_{2}=1.01$

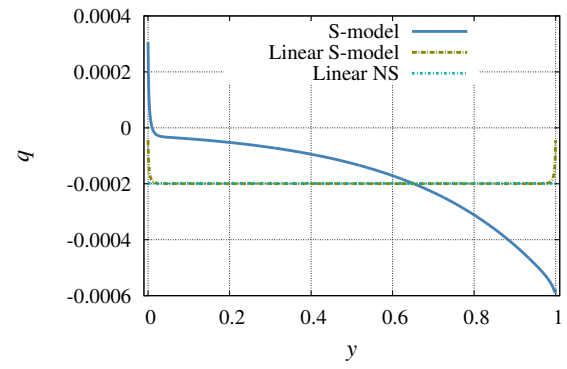

(d) $T_{1} / T_{2}=1.01, p_{1} / p_{2}=1.1$

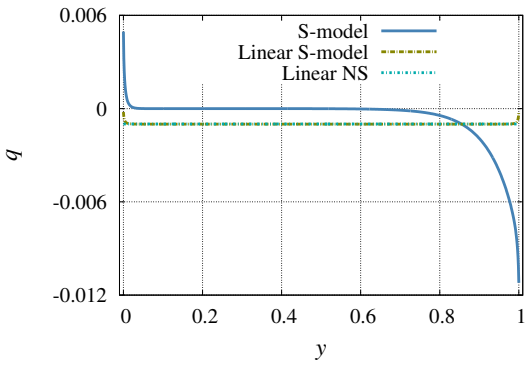

(f) $T_{1} / T_{2}=1.05, p_{1} / p_{2}=1.5$

Figure 9: Comparison of three results for $\delta=100$ and complete evaporation $\sigma=1$ : the numerical solution of the linearized S-model kinetic equation, the analytical solution of the linearized Navier-Stokes equations with the temperature and pressure jumps boundary conditions and the numerical solution of the non-linear S-model kinetic equation. The evaporation velocity and heat flux profiles in the gap are compared for three sets of saturation temperature and pressure ratios: (a), (b) $T_{1} / T_{2}=1.001, p_{1} / p_{2}=1.01$, (c), (d) $T_{1} / T_{2}=1.01, p_{1} / p_{2}=1.1$; (e), (f) $T_{1} / T_{2}=1.05, p_{1} / p_{2}=1.5$. 